TITLE:

\title{
Evaluation of dynamic tumour tracking radiotherapy with real- time monitoring for lung tumours using a gimbal mounted linac.
}

\section{AUTHOR(S):}

Matsuo, Yukinori; Ueki, Nami; Takayama, Kenji; Nakamura, Mitsuhiro; Miyabe, Yuki; Ishihara, Yoshitomo; Mukumoto, Nobutaka; ... Sawada, Akira; Kokubo, Masaki; Hiraoka, Masahiro

\section{CITATION:}

Matsuo, Yukinori ... [et al]. Evaluation of dynamic tumour tracking radiotherapy with real-time monitoring for lung tumours using a gimbal mounted linac.. Radiotherapy and oncology : journal of the European Society for Therapeutic Radiology and Oncology 2014, 112(3): 360-364

\section{ISSUE DATE:}

2014-09

URL:

http://hdl.handle.net/2433/192774

\section{RIGHT:}

(c) 2014 Elsevier Ireland Ltd.; この論文は出版社版でありません。引用の 際には出版社版をご確認ご利用ください。; This is not the published version. Please cite only the published version. 
Title:

2 Evaluation of dynamic tumour tracking radiotherapy with real-time monitoring for lung tumours using a

3 gimbal mounted linac

\section{Authors:}

6 Yukinori Matsuo, $\mathrm{MD}, \mathrm{PhD}^{1}$; Nami Ueki, MD, $\mathrm{PhD}^{1}$; Kenji Takayama, $\mathrm{MD}^{2}$; Mitsuhiro Nakamura,

$7 \quad \mathrm{PhD}^{1}$; Yuki Miyabe, $\mathrm{MS}^{1}$; Yoshitomo Ishihara, $\mathrm{MS}^{1}$; Nobutaka Mukumoto, $\mathrm{PhD}^{1}$; Shinsuke Yano, $\mathrm{RTT}^{3}$;

8 Hiroaki Tanabe, $\mathrm{MS}^{2}$; Shuji Kaneko, $\mathrm{BS}^{1,4}$; Takashi Mizowaki, MD, $\mathrm{PhD}^{1}$; Hajime Monzen, $\mathrm{PhD}^{1}$;

$9 \quad$ Akira Sawada, $\mathrm{PhD}^{5}$; Masaki Kokubo, $\mathrm{MD}, \mathrm{PhD}^{6}$; and Masahiro Hiraoka, $\mathrm{MD}, \mathrm{PhD}^{1}$.

\section{Affiliations:}

12 1. Department of Radiation Oncology and Image-applied Therapy, Kyoto University Graduate School of Medicine, Kyoto, Japan

14 2. Division of Radiation Oncology, Institute of Biomedical Research and Innovation, Kobe, Japan

15 3. Clinical Radiology Service Division, Kyoto University Hospital, Kyoto, Japan

16 4. Mitsubishi Heavy Industries, Ltd., Tokyo, Japan

17 5. Faculty of Medical Science, Kyoto College of Medical Science, Nantan, Japan 
18 6. Department of Radiation Oncology, Kobe City Medical Center General Hospital, Kobe, Japan

19

20 Corresponding author:

21 Yukinori Matsuo, MD, PhD.

22 Department of Radiation Oncology and Image-applied Therapy, Graduate School of Medicine, Kyoto

23 University, 54 Shogoin Kawahara-cho, Sakyo-ku, Kyoto, 606-8507, Japan.

24 Phone: +81-75-751-3762, fax: +81-75-771-9749

25 E-mail: ymatsuo@kuhp.kyoto-u.ac.jp

26

27 This paper consists of 21 pages and 2 tables. There are 2 supplementary figures.

28

29 Running head:

30

Dynamic tracking with real-time monitoring

31

32 Keywords: stereotactic body radiotherapy; lung cancer; dynamic tumour tracking; real-time monitoring;

33 feasibility study 
35

\section{Abstract:}

Purpose: To evaluate feasibility and acute toxicities after dynamic tumour tracking (DTT) irradiation with real-time monitoring for lung tumours using a gimbal mounted linac.

Materials and Methods: Spherical gold markers were placed around the tumour using a bronchoscope prior to treatment planning. Prescription dose at the isocentre was 56 Gy in 4 fractions for T2a lung cancer and metastatic tumour, and 48 Gy in 4 fractions for the others. Dose-volume metrics were compared between DTT and conventional static irradiation using in-house developed software.

Results: Of twenty patients enrolled, DTT radiotherapy was successfully performed for 16 patients, except 4 patients who coughed out the gold markers, one who showed spontaneous tumour regression, and one where the abdominal wall motion did not correlate with the tumour motion. Dose covering 95\% volume of GTV was not different between the two techniques, while normal lung volume receiving 20 Gy or more was reduced by $20 \%$. A mean treatment time per fraction was 36 minutes using DTT. With a median follow-up period of 13.2 months, no severe toxicity grade 3 or worse was observed.

Conclusions: DTT radiotherapy using a gimbal mounted linac was clinically feasible for lung treatment without any severe acute toxicity. 


\section{Introduction}

53 Lung cancer is the leading cause of cancer-related deaths in most countries, including the US[1] and

54 Japan[2]. There have been two trends in lung cancer in recent years. The first is the shift in the patient

55 population to older ages[1,2], and the second is the gradual increase in the ratio of early stage lung cancer[3,4]. Thus, development of a new treatment modality that is appropriate for elderly patients with

57 early stage lung cancer is desirable.

58 Stereotactic body radiotherapy (SBRT) was developed as a new treatment modality for early stage lung cancer in the late 1990s. Many retrospective studies and several multi-institutional prospective trials have demonstrated that excellent local control is obtained by SBRT, with acceptable toxicity[5-7]. Thus,

61 SBRT is now an important treatment option for patients with early stage non-small-cell lung cancer who

62 are medically inoperable and those who are elderly and relatively unfit for surgery.

63 The next innovation expected for lung SBRT is four-dimensional (4D) radiotherapy, which can cope

64 with tumour movement. The lung expands periodically according to respiration and a lung tumour moves mainly in the craniocaudal direction. The amplitude of craniocaudal tumour motion is around 1

$66 \mathrm{~cm}$ in mean, but can be 3-4 $\mathrm{cm}$ in some patients[8]. When the whole trajectory of a moving tumour is

67 included in the irradiation field, larger volumes of healthy tissues are irradiated. The latter leads to a risk 
69 irradiation technique that permits delivery of a dose to the tumour and limiting that to normal tissues by

70 coping with respiratory motion,may have the potential to improve the outcomes and to expand the

71 indications of SBRT.

72 The Vero4DRT (formerly called the MHI-TM2000; Mitsubishi Heavy Industries Ltd., Tokyo, Japan, and

73 BrainLab AG, Feldkirchen, Germany)[12] has two special features that allow dynamic tumour tracking

74 (DTT) with real-time monitoring. One is two sets of kilovoltage $(\mathrm{kV}) \mathrm{x}$-ray imagers, that can monitor the

75 three-dimensional position of the tumour in real-time via implanted fiducial markers, and the other is a

76 gimbal mounted linac, enabling DTT. Extensive evaluation of the characteristics of this DTT system

77 demonstrated high accuracies in treatment delivery to a moving target[13-17]. Previous experimental

78 validation offered the required confidence for clinical implementation of DTT treatment of lung tumours.

79 The purpose of this study was to evaluate feasibility and acute toxicities after DTT radiotherapy with

80 real-time monitoring in SBRT for lung cancers using the Vero4DRT system.

81

82 Materials and Methods

83 Patients

84 Eligibility criteria were as follows: (1) a single lung tumour with a diameter of $50 \mathrm{~mm}$ or less, (2) no

85 nodal or distant metastasis, (3) respiratory tumour movement of $5 \mathrm{~mm}$ or more, (4) age of 20 years or 
86 above, (5) performance status of 0-2, (6) arms could be held over the head for 30 min or more, (7) doses

87 to adjacent organs not exceeding the pre-determined constraints, which were the same as in the Japan

88 Clinical Oncology Group protocol 0403[18], and (8) written informed consent. This study was approved by the institutional review board. It was registered with the UMIN Clinical Trials Registry in Japan

(UMIN000005324).

91 internal surrogate for the tumour position. Treatment planning was carried out 1 week after insertion of the gold markers. At least 3 markers were required for the DTT irradiation to be performed.

97 On the simulation day, the patient was fixed in a supine position with both arms raised using an individualized vacuum pillow (BodyFIX; Elekta AB, Stockholm, Sweden, or ESFORM; Engineering System, Matsumoto, Japan). Ten respiratory phases of 4D CT were acquired in axial cine mode using a 
103 was sent to iPlan RT image (ver. 4.1; BrainLab AG) as a reference image. The coordinate for each phase

104 image from the 4D CT was modified so that its centroid of the fiducial markers was matched with that in

105 the breath-hold CT. Then, the 10 phase images were fused onto the breath-hold CT. After the CT scan,

106 the patient was moved to the Vero4DRT to perform a 4D modelling[14], which correlates the external

107 abdominal motion and the internal fiducial motion. The purpose of the modelling was to estimate the 4D

108 modelling error and the peak-to-peak amplitude of tumour motion.

110 Treatment planning

111 Gross tumour volumes (GTVs) were delineated on the breath-hold CT and 10 phase images. An internal

112 target volume for tracking (ITV) was defined as a composite of the eleven GTVs from the breath-hold

113 CT and the 10 phase images (Supplementary Fig. 1). Because the phase images were registered based on

114 the marker centroid, the ITV was supposed to compensate for tumour deformation and uncertainty in the

115 positional relationship between the tumour and fiducial makers during respiration[19]. Planning target

116 volume (PTV) for tracking was defined as the ITV plus setup error and additional margins to

117 compensate the 4D modeling error, baseline drift of the abdominal position, and mechanical errors of the

118 system. The setup error was estimated to be $2.5 \mathrm{~mm}$ in each direction as an inter-fraction positional

119 variation between the fiducials and the tumour[19]. A margin for the 4D modelling error was defined as 
120 a mean plus 2 times of standard deviation in the 4D model on the simulation day. A margin for the

121 baseline drift was estimated as $10 \%$ of the tumour motion amplitude. The mechanical errors were

122 defined as $0.5 \mathrm{~mm}[12]$. The PTV margin was defined as a linear sum of these errors. At least $5 \mathrm{~mm}$ was

123 required for the margin.

124 Monitor units (MUs) for the treatment beams were calculated using the X-ray voxel Monte Carlo

125 algorithm in iPlan RT dose (ver. 4.5.1; BrainLab AG) on the breath-hold exhale CT. The prescription

126 dose was 48 Gy in four fractions for stage IA lung cancer, and 56 Gy in four fractions for stage IB lung

127 cancer and metastatic lung tumour. This dose was prescribed at the isocentre. We typically arranged

128 seven beams: four non-coplanar and three coplanar beams. 6-MV x-ray beams were collimated to the

129 PTV plus 5-mm margin with a multi-leaf collimator. A static SBRT plan with non-tracking beams, based

130 on the motion-encompassing method[8], was also prepared as a backup if DTT irradiation could not be

131 achieved for some reason. Dose distributions were evaluated with an in-house developed software that

132 allows 4D dose calculation considering with the gimbal mounted linac[20]. The calculated dose

133 distributions for the 10 phase CT images were accumulated into the exhale breath-hold CT with

134 deformable image registration using MIM Maestro (ver. 5.2; MIM Software Inc., Cleveland, OH). If the

135 dose-volume metrics in the tracking plan were diagnosed to be superior to those in the static plan, the

patient underwent DTT irradiation. 


\section{Irradiation of treatment beams}

139 First, the patient was laid on the pre-formed vacuum pillow. Set-up error was corrected for bony

140 structures using the ExacTrac X-ray system. Second, a 4D model was built to correlate the infra-red

141 markers on the abdomen with the internal fiducial markers. Then, irradiation could start in dynamic

142 tracking mode with the beam following a location predicted by the 4D model based on the infra-red

143 markers placed on the patient's abdominal wall. During irradiation, the tumour and the internal fiducials

144 were monitored visually every second with EPID and the $\mathrm{kV}$ imagers. If the fiducial markers displaced

145 from the predicted positions by $3 \mathrm{~mm}$ or more in 3 consecutive frames on the $\mathrm{kV}$ images, the irradiation

146 was interrupted and rebuilding the 4D model was considered.

Follow-up after treatment

149 Follow-up visits were planned at 2, 4, 6, 9, and 12 months within the initial year after SBRT and every 3

150 months thereafter. A CT scan was performed at each visit. The follow-up period was defined as the

151 duration between the first day of treatment and the last follow-up visit or the date of death. Acute 


\section{Results}

Twenty-two patients were enrolled into this study between August 2011 and July 2013. No patients experienced toxicities related to the insertion of fiducial markers. Twenty-two of the 101 inserted markers were coughed out before the CT simulation. Consequently, the number of remaining makers in 4 patients decreased to two, which is insufficient for DTT to be performed. The planning procedures for DTT could not be performed in another two patients. One patient showed thoracic breathing that prevented the Vero system from tracking a tumour based on the abdominal motion (Supplementary Fig. regressed during 10 days between the fiducial insertion and the simulation. For the remaining 16 patients, the CT simulation for DTT was successfully performed. Characteristics of the 16 patients are shown in 
171 static plan in 5 patients. However, the difference did not exceed 0.3 Gy and was considered to be

172 clinically acceptable.

173 The DTT irradiation was completed for all 64 fractions for the 16 patients. Mean and standard deviation

174 in treatment time per fraction were 36.2 and 8.8 minutes (range, 19-70 minutes), respectively. The

175 treatment time exceeded 50 minutes in 5 fractions because of communication failures between the

176 tracking system and the gimbal system requiring a restart of the tracking procedure. The gold markers

177 were well recognized with $\mathrm{kV}$ x-ray imagers throughout all treatment fractions. The 4D modelling was

178 performed a mean of 1.9 times per fraction (range, 1 to 4 ).

179 With a median follow-up period of 13.2 months (range, 3.4-26.5 months), one patient experienced grade

1802 radiation pneumonitis. No severe toxicity grade 3 or worse has been observed in any of the patients.

181 Two patients died of cancer, and one died of infectious pneumonia. Local tumour control was achieved

182 except one patient who developed local recurrence at 12.0 months.

\section{Discussion}

185 The Vero4DRT system has two major advantages. The first is that tumour position can be monitored in

186 real time using $\mathrm{kV}$ imagers and an EPID. The second is that no extra treatment time over static SBRT

187 and no special training for breath control are needed for the 4D treatment, which is clinically beneficial 
188 both for patient comfort and the throughput of the treatment system.

189 Several uncertainties are associated with 4D irradiation, including tumour position prediction errors and

190 mechanical errors in beam delivery, which are problems to be overcome. In the Vero4DRT, kV imagers

191 can ensure that the positions of the tumour and fiducial markers correspond to the predicted sites. EPID

192 can be used to verify the tumour position in complement to the $\mathrm{kV}$ imagers[21]. Furthermore, log files

193 allow retrospective confirmation of the accuracy in the delivery of tumour tracking after treatment. Our

194 previous study, which evaluated the initial 10 patients from the present study cohort, confirmed high

195 accuracies in the tracking performance. The 95th percentiles of overall tracking errors were $1.3 \mathrm{~mm}, 2.4$

$196 \mathrm{~mm}$ and $1.4 \mathrm{~mm}$ in left-right, cranio-caudal and anterior-posterior directions, respectively[22].

197 The Task Group 76 of the American Association of Physicists in Medicine classified motion

198 management methods into five types: motion-encompassing, respiratory gating, breath-hold, forced

199 shallow breathing with abdominal compression, and real-time tumour-tracking methods[8]. The

200 motion-encompassing method is the most conventional and well-established. However, it uses the largest

201 field of the five methods, with a large PTV. The breath-hold technique and abdominal compression are

202 not always suitable for frail and elderly patients. Respiratory gating needs a longer treatment time than

203 motion-encompassing methods. With a dynamic tracking method, patients do not need to hold or limit

204 their breath, and the tumour is always irradiated by the treatment beams without intermittence, leading to 
205 a shorter treatment time than the breath-hold methods or the gating methods, and a comparable treatment

206 time to the motion-encompassing methods. Dynamic tumour tracking is considered to be the favourable

207 method among the five motion management methods from the patient compliance and comfort points of

208 view. According to a Japanese survey of SBRT in 2009[23], the most frequent response regarding the

209 time needed for a single daily fraction was $30 \mathrm{~min}$, followed by $40 \mathrm{~min}$. Our result of $36.2 \mathrm{~min}$ for a

210 single fraction was thus comparable with standard times for SBRT in Japan. In addition to patient

211 comfort, a few studies have suggested that prolongation of the treatment time for a single fraction may

212 reduce its biological effectiveness[24,25]. Unfortunately, considerable prolongation of treatment time

213 over 50 minutes occurred in 5 fractions in the present study. The cause for the prolongation was

214 immaturity of the tracking system including software. To realize DTT irradiation, the tracking software

215 needed to communicate with other systems in real time and to command several operations including

216 control of the gimbal motion, process of signals from the infra-red camera and analysis of images from

217 the $\mathrm{kV}$ x-ray imagers. The tracking system had initial instability in the communication process where the

218 system could not simultaneously execute such different tasks. Some adaptations to the tracking system

219 have been introduced after the present study with the aim of improving stability. Volumetric modulated

220 arc therapy, which can reduce total MUs and consequently reduce treatment time, is being applied to

221 SBRT for the lung[26]. Arc irradiation application to DTT is needed for reduction of treatment time with 
222 the Vero4DRT. The Vero4DRT achieved the same level of geometric and dosimetric accuracy with 223 conformal arc DTT irradiation as that with fixed-port DTT[27]. A treatment planning system capable of

224 VMAT plan is under development for the Vero4DRT.

225 The disadvantages of the current Vero4DRT system are its dependence on 4D modeling and fiducial

226 marker insertion. If no appropriate 4D model is acquired, dynamic tracking cannot be performed, as

227 occurred in one patient. One solution for this issue is to track a tumour based on real-time stereo

228 fluoroscopy without any 4D model. However, this method introduces another problem of skin doses due

229 to the continuous fluoroscopy during a treatment time. Another way to improve the 4D model is a

230 training to encourage for patients to perform periodical abdominal breathing[28].

231 The Vero4DRT currently requires fiducial markers with x-ray fluoroscopy monitoring to detect the

232 tumour position and create the 4D model. Two types of fiducials are applicable to the Vero4DRT: one is

233 a spherical marker, and the other is a cylindrical marker. We used the spherical gold markers that were

234 inserted into a peripheral bronchiole under bronchoscopy. This method was reported in more detail by

235 Imura et al. from Hokkaido University[29]. All 57 patients in their report tolerated the marker

236 implantation procedure and only one experienced pneumothorax, which resolved with bed rest. The

237 toxicity rate was much lower than those of percutaneous insertion methods[30,31]. Trade-offs, however,

238 for the reduced toxicity are marker dislocation between insertion and radiotherapy, and positional 
239

240

241

242

243

244

245

246

247

248

250

251

252

uncertainty between the tumour and makers. Imura et al. reported that $25 \%$ of the inserted markers could not be detected throughout the treatment period. Indeed, $22 \%$ of the markers in this study were coughed out before the CT simulation, and 4 of 22 (18\%) patients could not undergo the DTT due to the marker dislocation. The reason why the Vero4DRT system requires 3 markers or more in the use of spherical markers is to detect the marker dislocation. Regarding the positional uncertainty between the tumour and markers, Ueki et al. evaluated intra- and interfractional variations[19]. Root mean squares in the intrafractional variations were $0.6 \mathrm{~mm}, 0.9 \mathrm{~mm}$ and $1.5 \mathrm{~mm}$ in the right-left, anteroposterior and craniocaudal directions, respectively. Moderate correlations of the intrafractional variation with tumour motion amplitude and tumour-marker distance were observed with correlation coefficients of 0.5490.780. However, the intrafractional error did not always distribute symmetrically around zero, but often distributed in one side above or below zero. The direction and amplitude of the intrafractional variations varied between patients. Base on the results, we judged that a uniform isotropic margin was inadequate to cover the intrafractional variations, and that the fused CT approach as in the Method section was suitable for DTT planning. The interfractional variation could be covered by a 2.5-mm margin.

In conclusion, DTT radiotherapy with real-time monitoring using a gimbal mounted linac was clinically feasible for the lung without any severe acute toxicity. 


\section{Conflict of interests:}

257 T.M., M.K. and M.H. have consultancy agreements with Mitsubishi Heavy Industries Ltd., Japan. A sponsored research program is provided by Mitsubishi Heavy Industries Ltd.

\section{Funding resources:}

\section{References}

268 [1] Siegel R, Naishadham D, Jemal A. Cancer statistics, 2013. CA Cancer J Clin 2013;63:11-30.

269 [2] Foundation for Promotion of Cancer Research. Cancer Statistics in Japan-2013 Available at: http://ganjoho.jp/pro/statistics/en/backnumber/2013_en.html. Accessed January 10, 2014. registry study of 11,663 surgical cases in 2004: demographic and prognosis changes over decade. J 
274 [4] Morgensztern D, Ng SH, Gao F, Govindan R. Trends in stage distribution for patients with non-small cell lung cancer: a National Cancer Database survey. J Thorac Oncol 2010;5:29-33.

276

277

278

279

280

281

282

283
[5] Timmerman R, Paulus R, Galvin J, Michalski J, Straube W, Bradley J, et al. Stereotactic body radiation therapy for inoperable early stage lung cancer. JAMA 2010;303:1070-6.

[6] Nagata Y, Hiraoka M, Shibata T, Onishi H, Kokubo M, Karasawa K, et al. Stereotactic Body Radiation Therapy For T1N0M0 Non-small Cell Lung Cancer: First Report for Inoperable Population of a Phase II Trial by Japan Clinical Oncology Group (JCOG 0403). Int J Radiat Oncol Biol Phys 2012;84:S46.

[7] Nagata Y, Hiraoka M, Shibata T, Onishi H, Kokubo M, Karasawa K, et al. A Phase II Trial of Stereotactic Body Radiation Therapy for Operable T1N0M0 Non-small Cell Lung Cancer: Japan Clinical Oncology Group (JCOG0403). Int J Radiat Oncol Biol Phys 2010;78:S27-8.

[8] Keall PJ, Mageras GS, Balter JM, Emery RS, Forster KM, Jiang SB, et al. The management of respiratory motion in radiation oncology report of AAPM Task Group 76. Med Phys 2006;33:3874900.

[9] Matsuo Y, Shibuya K, Nakamura M, Narabayashi M, Sakanaka K, Ueki N, et al. Dose-volume metrics associated with radiation pneumonitis after stereotactic body radiation therapy for lung cancer. Int J Radiat Oncol Biol Phys 2012;83:e545-9.

[10] Guckenberger M, Baier K, Polat B, Richter A, Krieger T, Wilbert J, et al. Dose-response relationship for radiation-induced pneumonitis after pulmonary stereotactic body radiotherapy. Radiother Oncol 2010;97:65-70.

[11] Borst GR, Ishikawa M, Nijkamp J, Hauptmann M, Shirato H, Bengua G, et al. Radiation pneumonitis after hypofractionated radiotherapy: evaluation of the LQ(L) model and different dose parameters. Int J Radiat Oncol Biol Phys 2010;77:1596-603.

[12] Kamino Y, Takayama K, Kokubo M, Narita Y, Hirai E, Kawawda N, et al. Development of a four-dimensional image-guided radiotherapy system with a gimbaled X-ray head. Int J Radiat Oncol Biol Phys 2006;66:271-8. 
300

301

302

303

304

305

306

307

308

309

310

311

313

314

315

316

317

318

319

320

321

322

323

324
[13] Mukumoto N, Nakamura M, Sawada A, Takahashi K, Miyabe Y, Takayama K, et al. Positional accuracy of novel x-ray-image-based dynamic tumor-tracking irradiation using a gimbaled MV x-ray head of a Vero4DRT (MHI-TM2000). Med Phys 2012;39:6287-96.

[14] Mukumoto N, Nakamura M, Sawada A, Suzuki Y, Takahashi K, Miyabe Y, et al. Accuracy verification of infrared marker-based dynamic tumor-tracking irradiation using the gimbaled x-ray head of the Vero4DRT (MHI-TM2000). Med Phys 2013;40:041706.

[15] Depuydt T, Verellen D, Haas O, Gevaert T, Linthout N, Duchateau M, et al. Geometric accuracy of a novel gimbals based radiation therapy tumor tracking system. Radiother Oncol 2011;98:365-72.

[16] Nakamura M, Sawada A, Ishihara Y, Takayama K, Mizowaki T, Kaneko S, et al. Dosimetric characterization of a multileaf collimator for a new four-dimensional image-guided radiotherapy system with a gimbaled x-ray head, MHI-TM2000. Med Phys 2010;37:4684-91.

[17] Takayama K, Mizowaki T, Kokubo M, Kawada N, Nakayama H, Narita Y, et al. Initial validations for pursuing irradiation using a gimbals tracking system. Radiother Oncol 2009;93:45-9.

[18] Matsuo Y, Takayama K, Nagata Y, Kunieda E, Tateoka K, Ishizuka N, et al. Interinstitutional variations in planning for stereotactic body radiation therapy for lung cancer. Int J Radiat Oncol Biol Phys 2007;68:416-25.

[19] Ueki N, Matsuo Y, Nakamura M, Mukumoto N, Iizuka Y, Miyabe Y, et al. Intra- and interfractional variations in geometric arrangement between lung tumours and implanted markers. Radiother Oncol 2014;110:523-8.

[20] Ishihara Y, Sawada A, Nakamura M, Mukumoto N, Kaneko S, Takayama K, et al. Development of Monte Carlo Dose Calculation System for Tumor-Tracking Irradiation with a Gimbaled X-Ray Head. Med Phys 2011;38:3644.

[21] Poels K, Depuydt T, Verellen D, Engels B, Collen C, Heinrich S, et al. A complementary dual-modality verification for tumor tracking on a gimbaled linac system. Radiother Oncol 2013;109:469-74. 
325

326

327

328

329

330

331

332

333

334

335

336

337

338

339

340

341

342

343

344

345

346

347

348

349

[22] Mukumoto N, Nakamura M, Yamada M, Takahashi K, Tanabe H, Yano S, et al. Intrafractional tracking accuracy in infrared marker-based hybrid dynamic tumour-tracking irradiation with a gimballed linac. Radiother Oncol 2014;111:301-5.

[23] Nagata Y, Hiraoka M, Mizowaki T, Narita Y, Matsuo Y, Norihisa Y, et al. Survey of stereotactic body radiation therapy in Japan by the Japan 3-D Conformal External Beam Radiotherapy Group. Int J Radiat Oncol Biol Phys 2009;75:343-7.

[24] Fowler JF, Welsh JS, Howard SP. Loss of biological effect in prolonged fraction delivery. Int J Radiat Oncol Biol Phys 2004;59:242-9.

[25] Shibamoto Y, Otsuka S, Iwata H, Sugie C, Ogino H, Tomita N. Radiobiological evaluation of the radiation dose as used in high-precision radiotherapy: effect of prolonged delivery time and applicability of the linear-quadratic model. J Radiat Res 2012;53:1-9.

[26] Verbakel WF a R, Senan S, Cuijpers JP, Slotman BJ, Lagerwaard FJ. Rapid delivery of stereotactic radiotherapy for peripheral lung tumors using volumetric intensity-modulated arcs. Radiother Oncol 2009;93:122-4.

[27] Ono T, Miyabe Y, Yamada M, Shiinoki T, Sawada A, Kaneko S, et al. Geometric and dosimetric accuracy of dynamic tumor-tracking conformal arc irradiation with a gimbaled x-ray head. Med Phys 2014;41:031705.

[28] Nakamura M, Sawada A, Mukumoto N, Takahashi K, Mizowaki T, Kokubo M, et al. Effect of audio instruction on tracking errors using a four-dimensional image-guided radiotherapy system. J Appl Clin Med Phys 2013;14:255-64.

[29] Imura M, Yamazaki K, Shirato H, Onimaru R, Fujino M, Shimizu S, et al. Insertion and fixation of fiducial markers for setup and tracking of lung tumors in radiotherapy. Int J Radiat Oncol Biol Phys 2005;63:1442-7.

[30] Kothary N, Heit JJ, Louie JD, Kuo WT, Loo BW, Koong A, et al. Safety and efficacy of percutaneous fiducial marker implantation for image-guided radiation therapy. J Vasc Interv Radiol 2009;20:235-9. 
350

351

352

353

354

355

356

357

358

359

360

361

362

363

364

volume for the ITV.

\section{Figure legend:} tracking
[31] Hong JC, Yu Y, Rao AK, Dieterich S, Maxim PG, Le Q-T, et al. High retention and safety of percutaneously implanted endovascular embolization coils as fiducial markers for image-guided stereotactic ablative radiotherapy of pulmonary tumors. Int J Radiat Oncol Biol Phys 2011;81:85-90.

Supplementary figure 1. A schema for definition of an internal target volume (ITV) for

a) An example of intrafractional change in alignment of gross tumour volume (GTV) and fiducial markers, and intrafractional tumour deformation.

b) Definition of ITV for tracking. The GTVs are fused based on the marker centroids. Then, an ITV for tracking (the thick red line) is defined as a composite of the fused GTVs.

c) A screenshot from a case. Several lines with various colours are shown around the tumour, which indicate GTVs from phase images of 4D CT. An ITV (not shown) is defined as a volume including all the GTVs. The outermost pink line indicates a planning target 
367 The tumour position in the superior-inferior (SI) direction and the abdominal wall position

368 in the anterior-posterior (AP) direction are shown in blue and red lines, respectively. Note

369 that the patient showed thoracic breathing where the tumour moved without any

370 abdominal motion during the initial 10 seconds. Thoracic breathing was the dominant

371 respiratory pattern in the patient so that the Vero system could not create an appropriate

$3724 \mathrm{D}$ model for the patient. 


\section{- fiducial markers}

\& centroid of markers

GTV

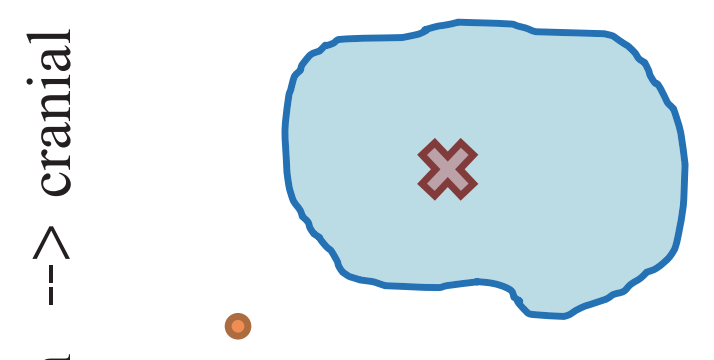

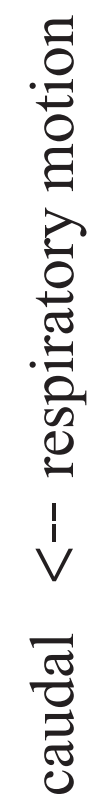

exhale

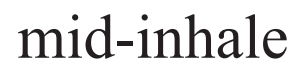

end-inhale

a) 


\section{ITV for tracking}

\section{$0 \infty$}

\section{0}

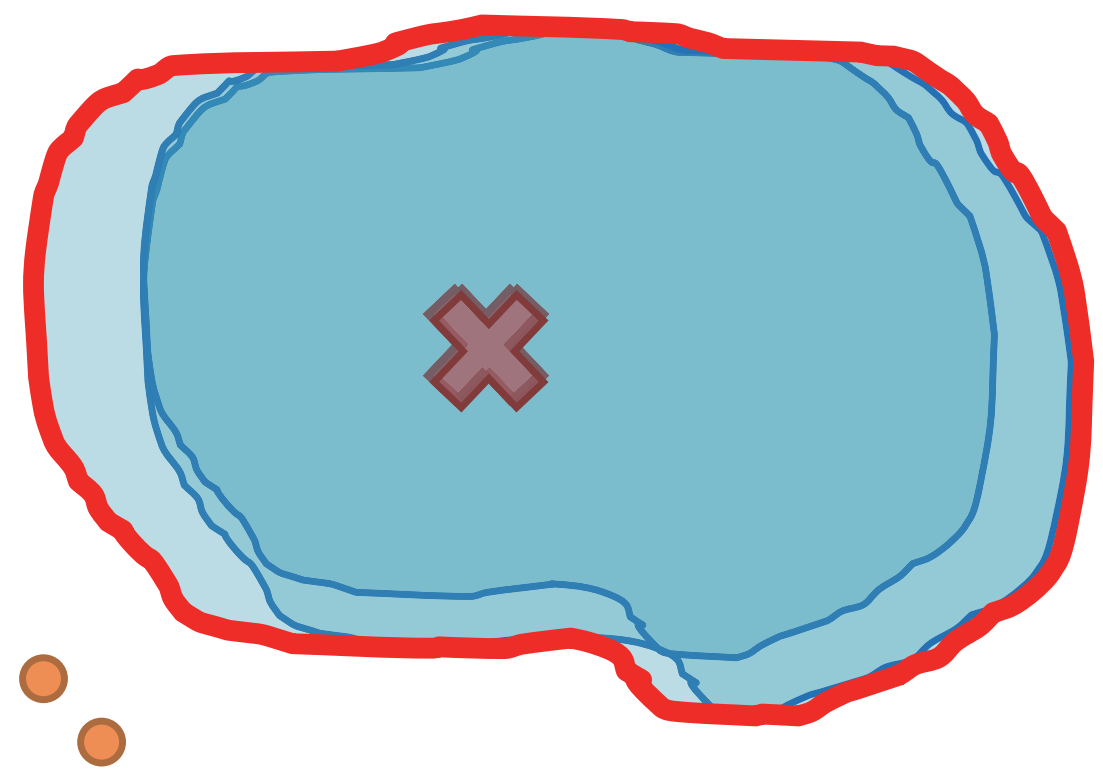

$8^{\circ}$ 


\section{3. 京都大学

KYOTO UNIVERSITY

A Self-archived copyin

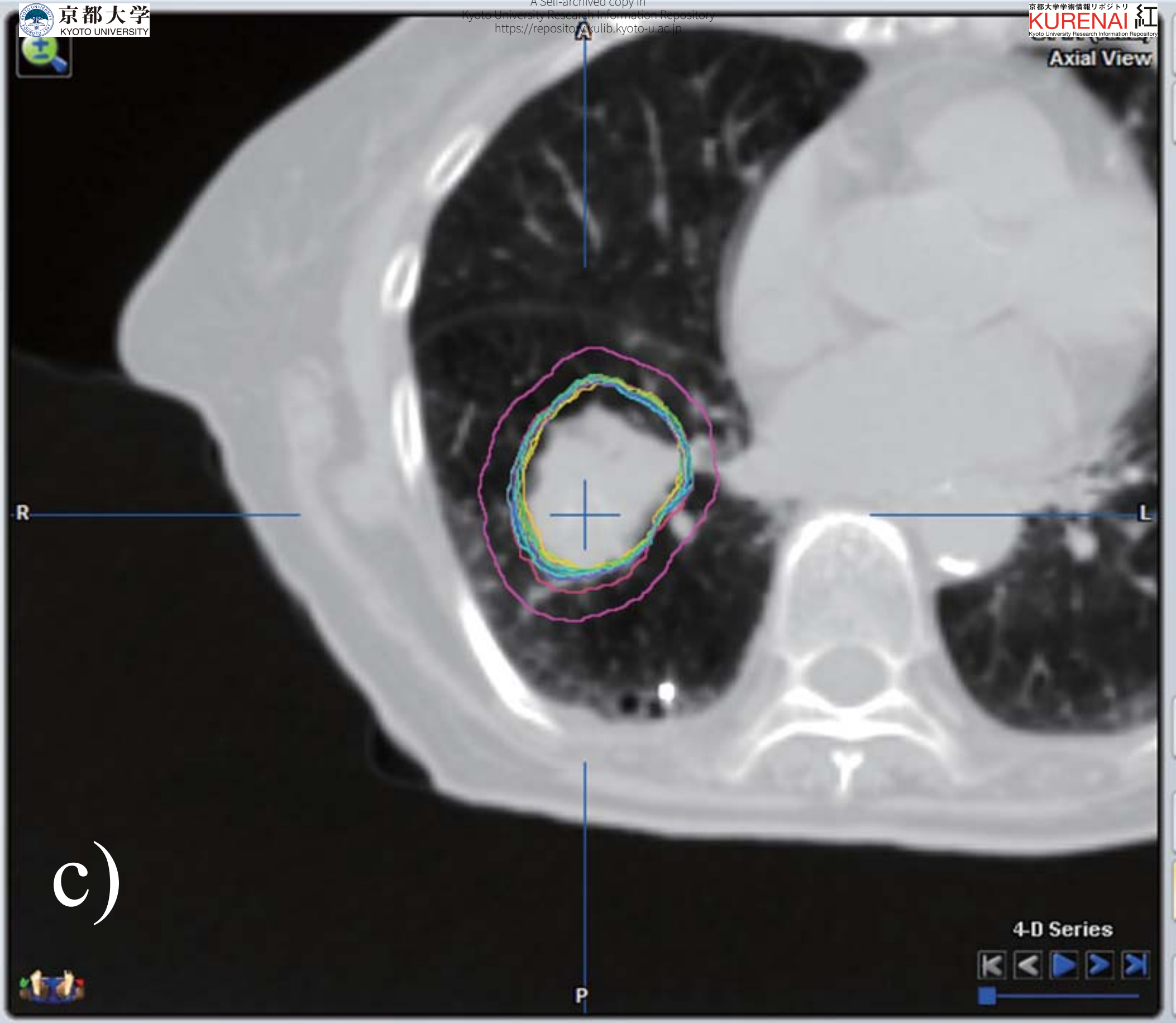

Es
$-$

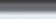$$
\text { ed copy in }
$$

-

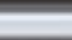

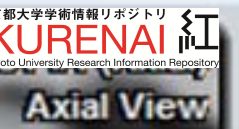

c)

of t:
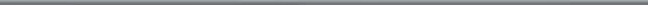


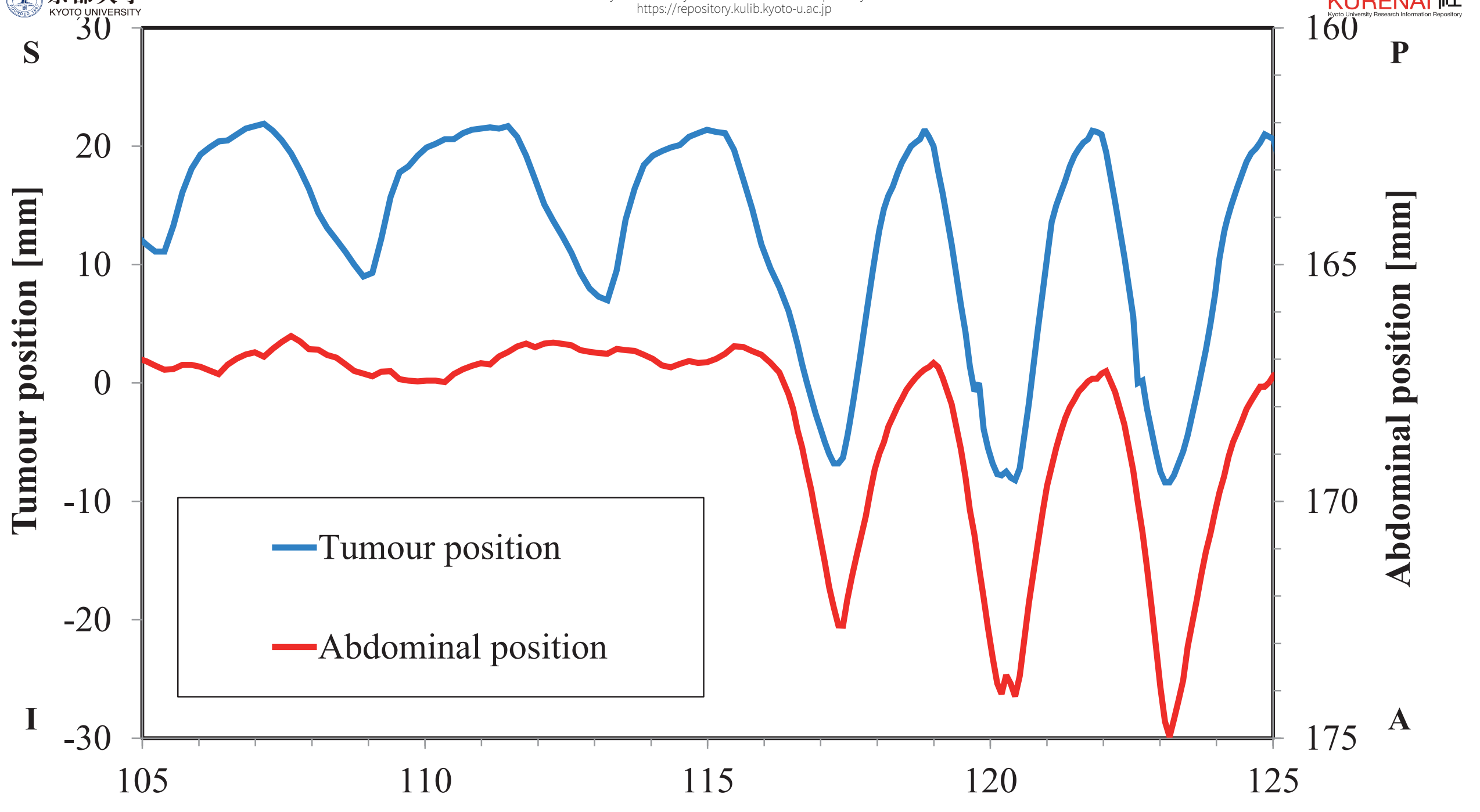

Time [sec] 